\title{
Large hole spin anticrossings in InAs/GaAs double quantum dots
}

\author{
F. Rajadell, J. I. Climente, and J. Planelles ${ }^{\text {a) }}$ \\ Departament de Química Física i Analítica, Universitat Jaume I, P.O. Box 224, E-12080 Castelló, Spain
}

(Received 9 August 2013; accepted 10 September 2013; published online 26 September 2013)

\begin{abstract}
We show that hole states in InAs/GaAs double quantum dots can exhibit spin anticrossings of up to $1 \mathrm{meV}$, according to simulations with a three dimensional Burt-Foreman Hamiltonian including strain and piezoelectric fields. The spin mixing originates in the valence band spin-orbit interaction plus the spatial symmetry breaking arising from misalignment between the dots and piezoelectric potential. The values we report are in better agreement with experiments than previous theoretical estimates and yield good prospects for efficient hole spin control. (C) 2013 AIP Publishing LLC. [http://dx.doi.org/10.1063/1.4823458]
\end{abstract}

There is current interest in using the spin of carriers confined in semiconductor quantum dots (QDs) for single spintronic, optoelectronic, and quantum information research. ${ }^{1-4}$ Self-assembled InAs/GaAs QDs have been particularly successful at this regard because they combine high optical activity, which enables precise optical preparation and read-out of the spin degrees of freedom, ${ }^{5,6}$ with moderately strong spin-orbit interaction (SOI), which provides an additional knob for spin control.

In general, the spin of electrons and holes in InAs QDs is a fairly good quantum number except in the vicinity of level crossings between states with orthogonal spins, ${ }^{7}$ where SOI or hyperfine interaction with the lattice nuclei mixes the two states, lifting the degeneracy and forming a spin anticrossing. The importance of spin anticrossings, also referred to as spin hot spots, ${ }^{3,8,9}$ lies in the fact that they lead to fast spin flips. For this reason they have been proposed and used for spin manipulation protocols (see, e.g., Refs. 10-12). A strong SOI is desirable to obtain large spin anticrossings, thereby enabling faster operations. In some protocols, large anticrossings are also convenient to enhance the fidelity of the operations. ${ }^{12}$

For electrons, spin anticrossings are mainly due to Rashba and Dresselhaus SOI. Takahashi et al. reported gaps of $70-160 \mu \mathrm{eV}$ between the $s$ and $p_{-}$orbitals of single InAs/GaAs QDs. ${ }^{13}$ Greilich et al. investigated spin anticrossings between $s$-shell singlet and triplet states of two electrons in vertically stacked double quantum dots (DQDs), obtaining gaps under $10 \mu \mathrm{eV} .{ }^{14}$ In the same work, it was observed that the corresponding gap for holes was $36 \mu \mathrm{eV}$, four times greater. This is due to the inherent valence band SOI, which is generally stronger than that of the conduction band. Indeed, Doty et al. observed spin anticrossings as large as $200 \mu \mathrm{eV}$ for holes tunneling in the neutral exciton states of some self-assembled DQD structures. ${ }^{15}$ Soon after, the same authors reported a gap of $400 \mu \mathrm{eV}$ on a similar system. ${ }^{12}$ This is the largest spin anticrossing observed in the $s$-shell of InAs QDs so far.

The origin of these large spin anticrossings was investigated by some of us in Ref. 15. It arises from a combination of valence band SOI plus strong spatial asymmetries. Separately, the QDs can be modeled as circular structures.

${ }^{\text {a)} E l e c t r o n i c ~ m a i l: ~ j o s e p . p l a n e l l e s @ u j i . e s ~}$
The SOI then couples the Bloch and envelope angular momenta, giving rise to a total azimuthal angular momentum which acts as a pseudo-spin. When the vertically stacked QDs are perfectly aligned, the pseudo-spin is still a valid quantum number. However, DQDs tend to present a certain amount of misalignment between the dots. This breaks the total angular momentum symmetry enabling pseudo-spin anticrossings. The greater the misalignment, the larger the anticrossing is. Considering lateral offsets of $5 \mathrm{~nm}$ between the dot centers-the largest value observed in cross-sectional scanning tunneling microscopy-our theory explained the $200 \mu \mathrm{eV}$ gap observed. However, there are still a number of open questions which call for further study.

First, experiments show two anticrossing gaps at different electric fields which, in general, are of similar magnitude. By contrast our model predicted asymmetric gaps and only one of them had comparable magnitude to the experiment. ${ }^{15}$ Second, more recent experiments evidenced anticrossing gaps as large as $400 \mu \mathrm{eV}$. In order to explain this value, we had to assume not only $5 \mathrm{~nm}$ lateral offset, which is possible but unusual, but also narrow QD diameters. ${ }^{12}$ One starts suspecting that the model misses additional sources of asymmetry, which make wide gaps more frequent than expected. Third, the qubit architecture proposed in Ref. 12 requires spin anticrossings to be as large as possible. One then wonders if it is possible to exceed $400 \mu \mathrm{eV}$ in realistic conditions.

In this work, we answer the questions above. To this end, we improve the theoretical model of Refs. 12 and 15 in several ways: (i) we use a Hamiltonian in Cartesian coordinates, which allows us to find the exact three-dimensional eigenstates without approximations; (ii) we include strain and piezoelectric fields; and (iii) we include the magnetic field making use of the minimal coupling prior to introducing the envelope function approximation, which has been shown to be more reliable for DQDs than the standard Luttinger model. ${ }^{16}$ We find that strain and piezoelectric fields provide an important contribution to hole spin anticrossings. The former enhances light hole (LH) delocalization in the interdot barrier, while the latter further breaks the angular symmetry. In combination with misalignment, this gives rise to improved agreement with the experiments. Besides, we predict anticrossing gaps can reach $1 \mathrm{meV}$ for DQDs with strong coupling and severe misalignment. 
The Hamiltonian we use to describe hole states reads

$$
\mathbf{H}=\mathbf{H}_{B F}+\mathbf{H}_{B}+\mathbf{H}_{\text {strain }}+\left(V(\vec{r})+e\left(\phi_{p z}(\vec{r})+F z\right)\right) \mathbf{I} .
$$

Here $\mathbf{H}_{B F}$ is the four-band Burt-Foreman Hamiltonian in Cartesian coordinates, which considers heavy hole-light hole (HH-LH) subband coupling and position-dependent effective masses. ${ }^{17} \mathbf{H}_{B}$ are the terms corresponding to a homogeneous magnetic field applied along the growth direction, $\mathbf{B}=(0,0, B)$, which we implement following Ref. 16. $\mathbf{H}_{\text {strain }}$ is the four-band Bir-Pikus Hamiltonian describing the effect of strain through deformation potentials. ${ }^{17} V(\vec{r})$ is the bandoffset potential, $e$ the hole charge, $\phi_{p z}$ the piezoelectric potential, $F$ an electric field applied along the growth direction and I a rank-4 identity matrix. Hamiltonian (1) is solved numerically after obtaining the strain tensors and piezoelectric fields using the Comsol package. ${ }^{18}$ The eigenstates are Luttinger spinors of the form

$$
\Psi_{h}=\sum_{J z=-3 / 2}^{3 / 2} f_{J_{z}}(\vec{r})\left|J=3 / 2, J_{z}\right\rangle,
$$

where $f_{J_{z}}(\vec{r})$ is the envelope function and $\left|J=3 / 2, J_{z}\right\rangle$ the periodic function with Bloch angular momentum $J_{z}$. $J_{z}= \pm 3 / 2$ corresponds to spin up and spin down HH components, while $J_{z}= \pm 1 / 2$ corresponds to LH components.

We study hole states confined in typical self-assembled InAs/GaAs DQD structures. Fig. 1(a) shows a schematic of the structure we consider. The QDs have truncated cone shape. Following Refs. 19 and 20, we assume trumpet-like alloy composition. Material parameters can be found in Ref. 18. Because the piezoelectric coefficient is influenced by strain, inside the QD we take the value proposed by Seguin et al., ${ }^{21} e_{14}=-0.385 \mathrm{C} / \mathrm{m}^{2} .{ }^{22}$ A magnetic field of $B=8 \mathrm{~T}$ is used in all calculations.

A prominent example of the spin anticrossings we study is illustrated in Fig. 1(b), which shows the low-energy hole states in a DQD with strong misalignment. One can see a series of anticrossings with increasing electric field, labeled as $\Delta_{1}^{c}, \Delta_{2}^{c}, \Delta_{1}^{s}$, and $\Delta_{2}^{s}$. To explain the origin of the anticrossings, we analyze the wave function of each energy level. In all cases, the dominant component of the Luttinger spinor is a HH. We use $\Uparrow(\Downarrow)$ to denote $J_{z}=+3 / 2(-3 / 2)$ and place the arrow in the upper or lower QD to represent the wave function localization. One can see that $\Delta_{1}^{c}$ and $\Delta_{2}^{c}$ are the usual charge transfer anticrossings, where $\mathrm{HH}$ localized in different dots hybridize to form molecular states all over the DQD before exchanging the location. ${ }^{23-26}$ By contrast, $\Delta_{1}^{s}$ and $\Delta_{2}^{s}$ are anticrossings between states with opposite $\mathrm{HH}$ spin and localization. The presence and, especially, the magnitude (hundreds of $\mu \mathrm{eV}$ ) of these anticrossings is surprising, because one does not expect coupling between spatially separated $\mathrm{HH}$ which on top have different spins (none of the terms in Hamiltonian (1) provides direct admixture between $J_{z}= \pm 3 / 2$ components).

It was shown in Ref. 15 that the spin anticrossings originate in the small LH components of the spinor. Because of their lighter mass, LH can be delocalized over the DQD even

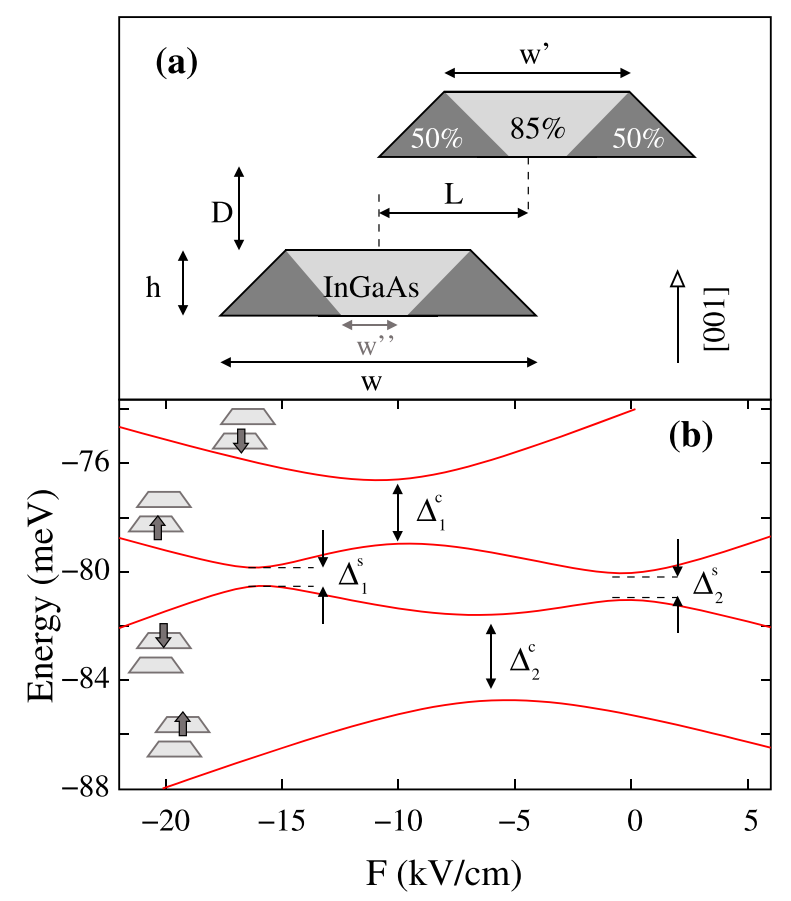

FIG. 1. (a) Schematic cross-section of the InAs/GaAs DQD structure studied in this paper. The percentages give the In composition in each region. Geometrical parameters: $h=3 \mathrm{~nm}, w=15 \mathrm{~nm}, w^{\prime}=9 \mathrm{~nm}, w^{\prime \prime}=2.7 \mathrm{~nm}$. Interdot barrier $D$ and lateral offset $L$ vary in each calculation. (b) Hole energy levels as a function of the electric field in a DQD with $D=3 \mathrm{~nm}$, $L=5 \mathrm{~nm}$ and magnetic field $B=8 \mathrm{~T}$. Energies are referred to the top of the valence band. The insets indicate the spin and the localization of the dominant $\mathrm{HH}$ component. Note the large magnitude of the spin anticrossing gaps, $\Delta_{1}^{s}$ and $\Delta_{2}^{s}$.

when $\mathrm{HH}$ are confined inside one of the dots, thus providing a channel for coupling. Besides, HH $J_{z}= \pm 3 / 2$ components interact in a second-order process through the $\mathrm{LH}\left(J_{z}= \pm 1 / 2\right)$ components (see $\mathbf{H}_{B F}$ and $\mathbf{H}_{\text {strain }}$ ). The magnitude of the spin anticrossing gaps is then proportional to (i) the strength of HH-LH coupling, (ii) the degree of LH delocalization, and (iii) the deviation from axial symmetry.

With the above rules in mind, we next investigate how different factors affect the magnitude of $\Delta_{1}^{s}$ and $\Delta_{2}^{s}$. We start by calculating the anticrossing gaps in DQDs with no strain or piezoelectric field in Fig. 2(a). It can be seen that both gaps increase with increasing lateral offset. This is due to the deviation from axial symmetry. However, $\Delta_{2}^{s}$ increases much faster than $\Delta_{1}^{s}$. This is essentially the same result as obtained with the model of Refs. 12 and 15, which is confirmed here using exact numerical solutions of the three-dimensional system. ${ }^{27-29}$ To understand the different magnitude of the gaps, note that $\Delta_{2}^{s}$ takes place at $F \sim 0$, while $\Delta_{1}^{s}$ does so at $F \ll 0$ (see the example in Fig. 1(b)). For the symmetric DQD we consider, at $F \sim 0$ the LH are near the homopolar molecular bond, while at $F \ll 0$ they are in the heteropolar regime. ${ }^{30}$ Therefore, LH delocalization is stronger at $F \sim 0$ and $\Delta_{2}^{s}>\Delta_{1}^{s}$. This is confirmed by inspecting the probability density functions of LH. A clear example is given in the insets of Fig. 2(a), which compare the LH probability at the electric fields of $\Delta_{1}^{s}$ (left panel) and $\Delta_{2}^{s}$ (right panel).

In a second step, we include strain in the Hamiltonian. The resulting gaps are plotted in Fig. 2(b). In general, the gaps still increase with the lateral offset, but there are two 


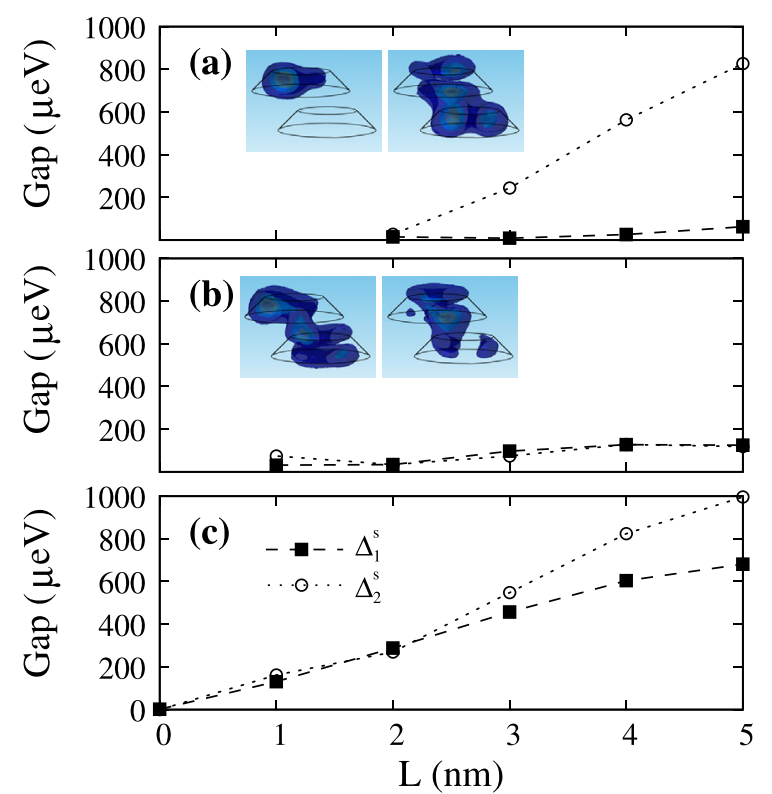

FIG. 2. Spin anticrossing gaps as a function of the lateral offset between QDs for a DQD with $D=3 \mathrm{~nm}$. (a) $\mathbf{H}_{\text {strain }}=0, \quad \phi_{p z}=0$. (b) $\mathbf{H}_{\text {strain }} \neq 0, \phi_{p z}=0$. (c) $\mathbf{H}_{\text {strain }} \neq 0, \phi_{p z} \neq 0$. Note that the inclusion of strain makes $\Delta_{1}^{s} \sim \Delta_{2}^{s}$ and that of $\phi_{p z}$ enhances the magnitude of the anticrossings. The insets in (a) and (b) show isosurfaces of the density probability of the $J_{z}=1 / 2$ (LH) component at the electric fields of $\Delta_{1}^{s}$ (left panel) and $\Delta_{2}^{s}$ (right panel) for the high-energy state of the anticrossing.

important differences: (i) the increase is lower than in the absence of strain and (ii) $\Delta_{1}^{s}$ and $\Delta_{2}^{s}$ are already of comparable magnitude. Both effects can be interpreted from the strain potential experienced by $\mathrm{LH}$, which is represented in the left column of Fig. 3. There, panels (a), (b), and (c) give an inplane cross-section of the LH strain potential in the middle of the upper dot, interdot barrier, and lower dot, respectively. One can see that the strain forms a potential well in between the dots. This enhances LH delocalization even for $F \ll 0$, as shown in the insets of Fig. 2(b), thus removing the big differences between $\Delta_{1}^{s}$ and $\Delta_{2}^{s}$. On the other hand, HH remains mainly localized inside the dots, ${ }^{31}$ so the overall HH-LH coupling is weakened. This explains the reduced magnitude of the spin anticrossings.

Last, we complete the Hamiltonian by including the piezoelectric field. The resulting gaps are plotted in Fig. 2(c). As compared to the previous systems, one concludes that the main effect of the piezoelectric field is to enhance the magnitude of the spin anticrossings. As a matter of fact, gaps of $600 \mu \mathrm{eV}$, the largest feasible value predicted in Ref. 12 calculations, are obtained with lateral offsets of only $L \approx 3 \mathrm{~nm}$, well below the $5 \mathrm{~nm}$ estimated in such work. What is more, for $L=5 \mathrm{~nm}$, the gaps we predict are as large as $1000 \mu \mathrm{eV}$, more than twice the largest value reported to date. The experimental realization of DQDs with such large anticrossing gaps would be an asset for current proposals to use the hole of spins for quantum information processing. ${ }^{12}$

The enhancement of the spin anticrossing gap by the piezoelectric field can be understood from the strongly asymmetric potential it creates. This is shown in the right column of Fig. 3. As for the strain before, panels (d), (e), and (f) give an in-plane cross-section of the piezoelectric potential in the middle of the upper dot, interdot barrier, and lower dot. Clearly, the potential lowers the angular symmetry in all

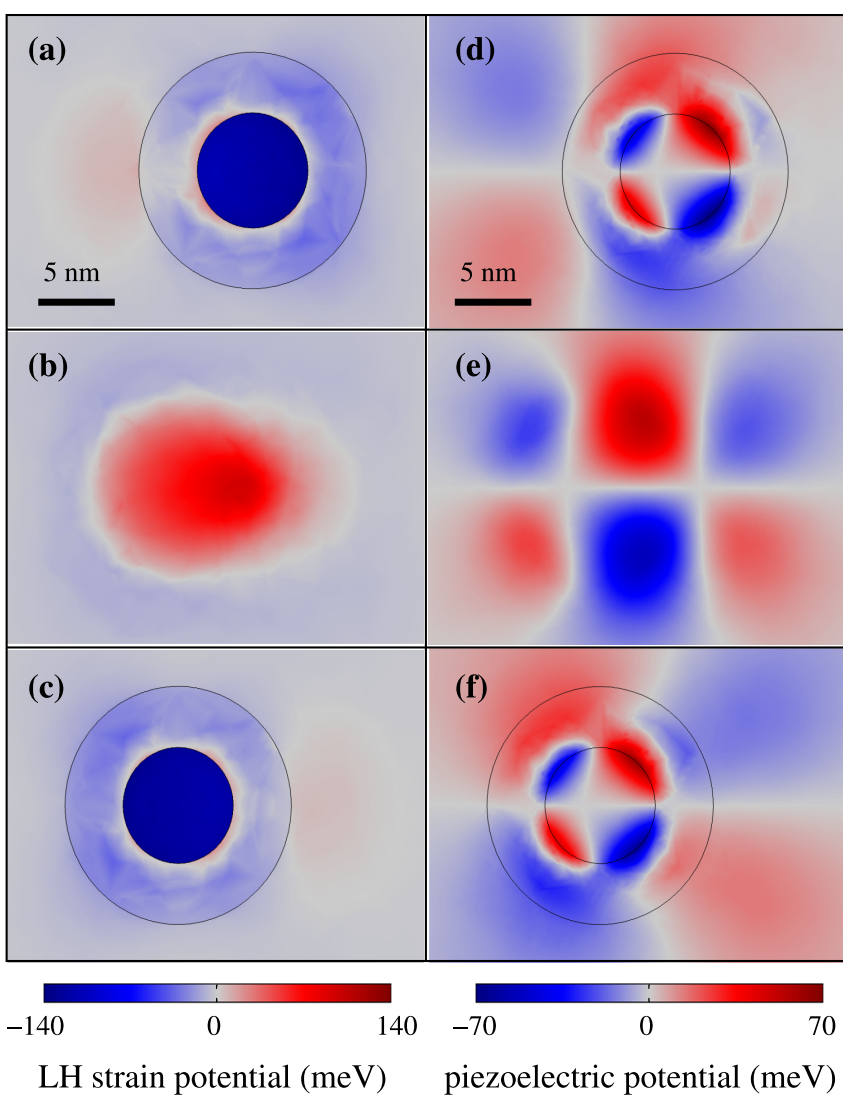

FIG. 3. In-plane cross-sections of the strain potential felt by the LH (a)-(c) and the piezoelectric potential (d)-(f) in a DQD with $D=3 \mathrm{~nm}$ and $L=4 \mathrm{~nm}$. Top (bottom) panels show the potential at half height of the top (bottom) QD, while the middle panel shows that at half interdot barrier.

instances, thereby stimulating mixing of hole states which would otherwise have different total angular momentum. Yet, it is worth noting that misalignment still plays a central role in determining the gaps. For $L=0$, both $\Delta_{1}^{s}$ and $\Delta_{2}^{s}$ are negligible in spite of the piezoelectric field (see Fig. 2(c)). As a matter of fact, the strong piezoelectric potential in the barrier -see panel (e)-, which is in sharp contrast with that of aligned DQDs, ${ }^{32}$ is a consequence of misalignment.

To complete our study, we investigate the influence of the interdot barrier thickness on the spin anticrossings. Fig. 4 shows the gaps for DQDs with fixed lateral offset $(L=3 \mathrm{~nm})$ and varying $D$. The behavior is non-simple because of the presence of several competing factors. However, the general trend is clear: the spin anticrossing gaps tend to decrease with the barrier thickness. This is due to the weaker tunneling, which reduces LH delocalization.

In summary, we have shown that the large spin anticrossing gaps observed in $\mathrm{p}$-doped InAs/GaAs DQDs are due

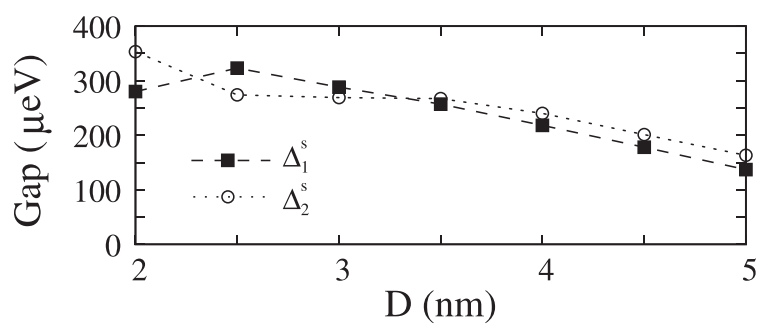

FIG. 4. Spin anticrossing gaps as a function of the interdot barrier thickness in a DQD with $L=3 \mathrm{~nm}$. 
to the combination of HH-LH coupling and the breaking of the envelope angular symmetry. The angular symmetry is lifted by misalignment and piezoelectric fields. In turn, the spin admixture, which is mainly provided by delocalized LH, is stimulated by strain and thin interdot barriers. Strain is also responsible for the experimental observation of pairs of gaps with similar magnitude.

We predict spin anticrossing gaps of up to $1 \mathrm{meV}$ in DQDs with strong tunneling and pronounced misalignment. Such large values imply fast spin-flip times and high fidelity spin control, which is encouraging for current endeavour to use the spin of holes in quantum information processing.

We thank M. F. Doty for helpful comments. Support from MINECO project CTQ2011-27324 and UJI-Bancaixa project P1-1B2011-01 is acknowledged.

${ }^{1}$ I. Zutic, J. Fabian, and S. Das Sarma, Rev. Mod. Phys. 76, 323 (2004).

${ }^{2}$ R. Hanson, L. P. Kouwenhoven, J. R. Petta, S. Tarucha, and L. M. K. Vandersypen, Rev. Mod. Phys. 79, 1217 (2007).

${ }^{3}$ E. N. Bulgakov and A. F. Sadreev, JETP Lett. 73, 505 (2001).

${ }^{4}$ J. Fischer, M. Trif, W. A. Coish, and D. Loss, Solid State Commun. 149 1443 (2009).

${ }^{5}$ A. N. Vamivakas, C.-Y. Lu, C. Matthiesen, Y. Zhao, S. Fält, A. Badolato, and M. Atatüre, Nature (London) 467, 297 (2010) and references therein.

${ }^{6}$ B. D. Gerardot, D. Brunner, P. A. Dalgarno, P. Öhberg, S. Seidl, M. Kroner, K. Karrai, N. G. Stoltz, P. M. Petroff, and R. Warburton, Nature (London) 451, 441 (2008).

${ }^{7}$ By hole spin we actually refer to the total angular momentum z-projection, which behaves as a pseudo-spin (see later). This is a usual simplification in the literature (Refs. 4, 6, 12,14, 15, and 23).

${ }^{8}$ P. Stano and J. Fabian, Phys. Rev. B 72, 155410 (2005).

${ }^{9}$ A. Bagga, P. Pietiläinen, and T. Chakraborty, Phys. Rev. B 74, 033313 (2006).

${ }^{10}$ J. M. Taylor, J. R. Petta, A. C. Johnson, A. Yacoby, C. M. Marcus, and M. D. Lukin, Phys. Rev. B 76, 035315 (2007).

${ }^{11}$ D. Kim, S. E. Economou, S. C. Badescu, M. Scheibner, A. S. Bracker, M. Bashkansky, T. L. Reinecke, and D. Gammon, Phys. Rev. Lett. 101, 236804 (2008).

${ }^{12}$ S. E. Economou, J. I. Climente, A. Badolato, A. S. Bracker, D. Gammon, and M. F. Doty, Phys. Rev. B 86, 085319 (2012).
${ }^{13}$ S. Takahashi, R. S. Deacon, K. Yoshida, A. Oiwa, K. Shibata, K. Hirakawa, Y. Tokura, and S. Tarucha, Phys. Rev. Lett. 104, 246801 (2010).

${ }^{14}$ A. Greilich, S. C. Badescu, D. Kim, A. S. Bracker, and D. Gammon, Phys. Rev. Lett. 110, 117402 (2013).

${ }^{15}$ M. F. Doty, J. I. Climente, A. Greilich, M. Yakes, A. S. Bracker, and D. Gammon, Phys. Rev. B 81, 035308 (2010).

${ }^{16} \mathrm{~J}$. Planelles and J. I. Climente, e-print arXiv:1303.6538.

${ }^{17}$ L. C. L. Y. Voon and M. Willatzen, The k.p Method (Springer, Berlin, 2009).

${ }^{18}$ See supplementary material at http://dx.doi.org/10.1063/1.4823458 for material parameters and computational details.

${ }^{19}$ M. A. Migliorato, A. G. Cullis, M. Fearn, and J. H. Jefferson, Phys. Rev. B 65, 115316 (2002).

${ }^{20}$ J. J. Finley, M. Sabathil, P. Vogl, G. Abstreiter, R. Oulton, A. I. Tartakovskii, D. J. Mowbray, M. S. Skolnick, S. L. Liew, A. G. Cullis, and M. Hopkinson, Phys. Rev. B 70, 201308(R) (2004).

${ }^{21}$ R. Seguin, A. Schliwa, S. Rodt, K. Pötschke, U. W. Pohl, and D. Bimberg, Phys. Rev. Lett. 95, 257402 (2005).

${ }^{22}$ Taking a bulk value, like $e=-0.045 \mathrm{C} / \mathrm{m}^{2}$, does not change the qualitative results but reduces the gaps by $\sim 40 \%$.

${ }^{23}$ E. A. Stinaff, M. Schneibner, A. S. Bracker, I. V. Ponomarev, V. L. Korenev, M. E. Ware, M. F. Doty, T. L. Reinecke, and D. Gammon, Science 311, 636 (2006).

${ }^{24}$ M. F. Doty, J. I. Climente, M. Korkusinski, M. Scheibner, A. S. Bracker, P. Hawrylak, and D. Gammon, Phys. Rev. Lett. 102, 047401 (2009).

${ }^{25}$ A. S. Bracker, M. Scheibner, M. F. Doty, E. A. Stinaff, I. V. Ponomarev, J. C. Kim, L. J. Whitman, T. L. Reinecke, and D. Gammon, Appl. Phys. Lett. 89, 233110 (2006).

${ }^{26}$ The charge transfer anticrossings in Fig. 1(b) are quite large $(\sim 3 \mathrm{meV})$ in spite of the heavy mass of holes because we consider a thin interdot barrier. There is experimental evidence for $\Delta^{c}>1 \mathrm{meV}$ for thin barriers in Refs. 24 and 25.

${ }^{27} \mathrm{We}$ have checked that including the leading term of valence band Rashba $\mathrm{SOI}^{28}$ barely increases $\Delta_{1}^{S}$ in spite of the strong electric field. The effect of cubic Dresselhaus SOI is a subject of future study. Its influence on the HHLH admixture is weaker than that coming from the quadratic valence band SOI considered here, ${ }^{29}$ but the simultaneous presence might enhance the gaps.

${ }^{28} \mathrm{R}$. Winkler, Spin-Orbit Coupling Effects in Two-Dimensional Electron and Hole Systems, (Springer, Berlin, 2003).

${ }^{29}$ J. I. Climente, C. Segarra, and J. Planelles, New J. Phys. 15, 093009 (2013).

${ }^{30}$ Note that the HH homopolar region differs from that of LH, owing to the different mass. It takes place at the fields where $\Delta_{1}^{c}$ and $\Delta_{2}^{c}$ appear.

${ }^{31}$ J. Planelles, J. I. Climente, F. Rajadell, M. F. Doty, A. S. Bracker, and D. Gammon, Phys. Rev. B 82, 155307 (2010).

${ }^{32}$ T. Andlauer and P. Vogl, Phys. Rev. B 79, 045307 (2009). 